\title{
Full-Round Differential Attack on the Original Version of the Hash Function Proposed at PKC'98
}

\author{
Donghoon Chang ${ }^{1 \star}$, Jaechul Sung ${ }^{2}$, Soohak Sung ${ }^{3}$, Sangjin Lee ${ }^{1}$, and \\ Jongin $\operatorname{Lim}^{1}$ \\ 1 Center for Information Security Technologies(CIST), \\ Korea University, Anam Dong, Sungbuk Gu, Seoul, Korea \\ \{pointchang, sangjin, jilim\}@cist.korea.ac.kr \\ 2 Korea Information Security Agency(KISA), \\ Karag-dong, Songpa-gu, Seoul, Korea \\ sjames@kisa.or.kr \\ 3 Paichai University, Daejeon, Korea, \\ sungsh@mail.paichai.ac.kr
}

\begin{abstract}
Shin et al. 4 proposed a new hash function with 160-bit output length at PKC'98. Recently, at FSE 2002, Han et al.5] cryptanalyzed the hash function proposed at PKC'98 and suggested a method finding a collision pair with probability $2^{-30}$, supposing that boolean functions satisfy the SAC(Strict Avalanche Criterion). This paper improves their attack and shows that we can find a collision pair from the original version of the hash function with probability $2^{-37.13}$ through the improved method. Furthermore we point out a weakness of the function comes from shift values dependent on message.
\end{abstract}

\section{Introduction}

MD4, MD5, RIPEMD-160, HAVAL, SHA-1 are well known dedicated hash functions. Dobbertin 2] 3] showed that there are serious weakness in MD4 and MD5. Haval 11 was attacked partially. Shin et al. 4 proposed a new hash function with 160-bit output length at PKC'98.

Recently, at FSE 2002, Han et al.[5] pointed out that, unlike the designer's attention, some of the boolean functions of the hash function proposed at PKC'98 do not satisfy the SAC(Strict Avalanche Criterion). And they analyzed the hash function proposed at PKC'98 and found a collision pair with probability $2^{-30}$, supposing that the boolean functions satisfy the SAC. However only one of boolean functions used in the hash function satisfies the SAC. So, their attack introduced at FSE 2002 can not be applied to the hash function itself.

This paper improves the method proposed at FSE 2002 and shows that we can find a collision pair "from the original version" of the hash function with

\footnotetext{
^ This work was supported by both Ministry of Information and Communication and Korea Information Security Agency, Korea, under project 2002-130
} 
probability $2^{-37.13}$ through the improved method. And we point out the problem of the hash function comes from shift values dependent on message. Next, we show that reduced versions for 3-pass HAVAL are attacked by our attack method.

\section{The Hash Function Proposed at PKC'98}

In this section, we briefly describe the hash function proposed at PKC'98, and we introduce notations used in this paper.

\begin{tabular}{|c||c|}
\hline word & 32-bit string \\
\hline block & 512-bit string used as input of compression function in the hash function \\
\hline+ & addition modulo $2^{32}$ operation between two words \\
\hline$X<<s$ & left rotation $\mathrm{X}$ by s bits \\
\hline $\mathrm{X} \wedge \mathrm{Y}$ & bitwise logical AND operation of $\mathrm{X}$ and $\mathrm{Y}$ \\
\hline $\mathrm{X} \vee \mathrm{Y}$ & bitwise OR operation of $\mathrm{X}$ and $\mathrm{Y}$ \\
\hline $\mathrm{X} \oplus \mathrm{Y}$ & bitwise XOR operation of $\mathrm{X}$ and $\mathrm{Y}$ \\
\hline
\end{tabular}

\subsection{Input Block Length and Padding}

An input message is processed by 512-bit block. The proposed hash function pads a message by appending a single bit 1 next to the least significant bit of the message, followed by zero or more bit 0s until the length of the message is 448 modulo 512, and then appends to the message the 64-bit original message length modulo $2^{64}$.

\subsection{Initial Value(IV)}

The initial values of five chaining variables $(A, B, C, D, E)$ used in processing message are as follows.

\begin{tabular}{|c|c|c|c|c|}
\hline $\mathrm{A}$ & $\mathrm{B}$ & $\mathrm{C}$ & $\mathrm{D}$ & $\mathrm{E}$ \\
\hline 0x67452301 & 0xefcdab89 & 0x98badcef & 0x10325476 & 0xc3d2e1f0 \\
\hline
\end{tabular}

\subsection{Constants}

The following numbers are used as constants $\left(K_{i}\right.$ is used in round $\left.i\right)$.

$$
K_{1}=0 \quad K_{2}=0 x 5 a 827999 K_{3}=0 x 6 e d 9 \mathrm{eba1} K_{4}=0 x 8 f 1 \mathrm{bbcdc}
$$

\subsection{Expansion of Message Variables}

Eight message variables, $X_{16}, X_{17}, \cdots, X_{23}$ are additionally generated from original sixteen input message words, $X_{0}, X_{1}, \cdots, X_{15}$ as follows. So twenty-four message words are applied to the compression function.

$$
X_{16+i}=\left(X_{0+i} \oplus X_{2+i} \oplus X_{7+i} \oplus X_{12+i}\right)^{<<1} \quad(i=0,1, \cdots, 7)
$$




\subsection{Ordering of Message Words}

This hash function consists of four rounds. Each round has 24 steps.

Table 1. The definition of permutation $\rho$

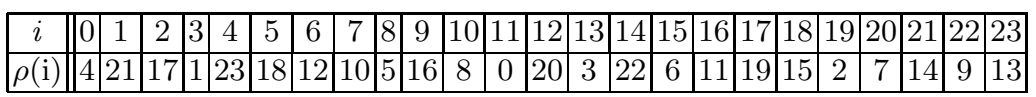

The ordering of message words is determined by $\rho$ as follows.

\begin{tabular}{|c||c|c|c|c|}
\hline Round & 1 & 2 & 3 & 4 \\
\hline Permutation & $i d$ & $\rho$ & $\rho^{2}$ & $\rho^{3}$ \\
\hline
\end{tabular}

\subsection{Boolean Functions}

The boolean functions used at each round are as follows. Only the function $f_{2}$ satisfies the SAC, while it is not the case for $f_{0}$ and $f_{1}$.

$$
\begin{aligned}
& f_{0}\left(x_{1}, x_{2}, x_{3}, x_{4}, x_{5}\right)=\left(x_{1} \wedge x_{2}\right) \oplus\left(x_{3} \wedge x_{4}\right) \oplus\left(x_{2} \wedge x_{3} \wedge x_{4}\right) \oplus x_{5} \\
& f_{1}\left(x_{1}, x_{2}, x_{3}, x_{4}, x_{5}\right)=x_{2} \oplus\left(\left(x_{4} \wedge x_{5}\right) \vee\left(x_{1} \wedge x_{3}\right)\right) \\
& f_{2}\left(x_{1}, x_{2}, x_{3}, x_{4}, x_{5}\right)=x_{1} \oplus\left(x_{2} \wedge\left(x_{1} \oplus x_{4}\right)\right) \oplus\left(\left(\left(x_{1} \wedge x_{4}\right) \oplus x_{3}\right) \wedge x_{5}\right)
\end{aligned}
$$

\subsection{Operation in One Step}

The operation in one step is defined as follows. The function $f_{0}$ is applied to the first round(0 23 step), the function $f_{1}$ is applied to the second round(24 47 step), the function $f_{2}$ is applied to the third round(48 71 step) and the function $f_{1}$ is applied to the fourth round(72 95 step).

$$
\begin{gathered}
A_{i}=\left(f\left(A_{i}, B_{i}, C_{i}, D_{i}, E_{i}\right)+X+K\right)^{<<S_{i}}, \quad B_{i}=B_{i}^{<<10} \\
A_{i+1}=E_{i}, B_{i+1}=A_{i}, C_{i+1}=B_{i}, D_{i+1}=C_{i}, E_{i+1}=D_{i}
\end{gathered}
$$

The operation in one step can be described in the figure 1 .

\subsection{Shift Operation}

The shift values used in each step, $S_{i}(i=0,1,2, \cdots, 95)$, are determined depending on message words as follows.

$$
S_{i}=X_{R(i \bmod 24)} \bmod 32
$$

Next table shows R function per round.

\begin{tabular}{|c||c|c|c|c|}
\hline Round & 1 & 2 & 3 & 4 \\
\hline R function & $\rho^{3}$ & $\rho^{2}$ & $\rho$ & $i d$ \\
\hline
\end{tabular}

For example, let's solve $S_{20}$, shift value at step 20 . The step 20 is in the first round, so R function is $\rho^{3}$. Therefore $S_{20}=X_{\rho^{3}(20)} \bmod 32=X_{8} \bmod 32$. 
Fig. 1. The operation in one step

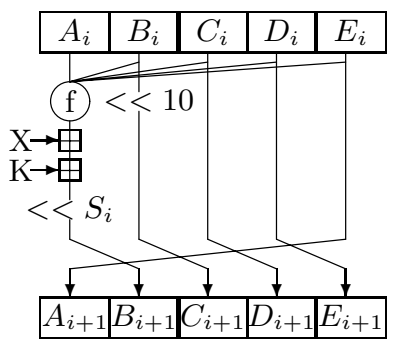

\subsection{Each Step Operation through the Table}

The following table shows each step operation from step 0 to step 6 . $A_{i}, B_{i}, C_{i}, D_{i}, E_{i}$ mean chaining variables after a equation (5) and before a equation (6) at $i$ step.

Table 2. The message input order, shift value and chaining values per step

\begin{tabular}{|c||ccccc|c|c|}
\hline \multicolumn{1}{|c||}{ step } & $\mathrm{A}$ & $\mathrm{B}$ & $\mathrm{C}$ & $\mathrm{D}$ & $\mathrm{E}$ & Input & Shift value(mod 32) \\
\hline \hline 0 & $A_{0}$ & $B_{0}$ & \multicolumn{1}{l|}{$C_{0}$} & \multicolumn{1}{l|}{$D_{0}$} & $E_{0}$ & $X_{0}$ & $X_{13}$ \\
\hline 1 & $A_{1}$ & $B_{1}$ & $C_{1}$ & $D_{1}$ & $E_{1}$ & $X_{1}$ & $X_{22}$ \\
\hline 2 & $A_{2}$ & $B_{2}$ & $C_{2}$ & $D_{2}$ & $E_{2}$ & $X_{2}$ & $X_{2}$ \\
\hline 3 & $A_{3}$ & $B_{3}$ & $C_{3}$ & $D_{3}$ & $E_{3}$ & $X_{3}$ & $X_{14}$ \\
\hline 4 & $A_{4}$ & $B_{4}$ & $C_{4}$ & $D_{4}$ & $E_{4}$ & $X_{4}$ & $X_{3}$ \\
\hline 5 & $A_{5}$ & $B_{5}$ & $C_{5}$ & $D_{5}$ & $E_{5}$ & $X_{5}$ & $X_{6}$ \\
\hline 6 & $A_{6}$ & $B_{6}$ & $C_{6}$ & $D_{6}$ & $E_{6}$ & $X_{6}$ & $X_{7}$ \\
\hline
\end{tabular}

$\square$ : The updated part per step

\section{The Analysis of the Original Version of the Hash Function Proposed at PKC'98}

\subsection{The Analysis of Input and Output Difference for Boolean Functions}

Boolean function $f_{2}$ satisfies the SAC. This means that when it has a difference at only one bit, output bit becomes zero with $1 / 2$ probability. At FSE 2002, under supposing that three boolean functions satisfy the SAC, Han et al. proposed a method finding a collision pair with probability $2^{-30}$. But in fact, boolean functions $f_{0}$ and $f_{1}$ do not satisfy the SAC. In case of $f_{0}$, when only last input 
bit has a difference, output difference becomes always 1(table 3-5). Also, in case of $f_{1}$, when only second input bit has a difference, output difference becomes always 1(table 3-13). These mean the followings: if a message word having a difference is an input of a round using the function $f_{0}$, the difference avalanche occurs after four steps because fifth chaining variable have a difference. If message word having a difference is input of a round using the function $f_{1}$, the difference avalanche occurs after one step because second chaining variable have a difference. And the effect of difference avalanche is larger according as the number of steps increases. Therefore the method proposed by Han et al. at FSE 2002 cannot be applied to the original version of the hash function. But, in case of $f_{0}$, if we give simultaneously differences to fifth chaining variable and other chaining variable, the output bit becomes zero with some probability(table 3$8,9,11)$. This is similar to $f_{1}$. We will use this fact to attack the original version of the hash function proposed at PKC'98.

Table 3. The probability that output difference bit becomes 0 with the change of location of input difference bit of boolean function $f_{i}$

\begin{tabular}{|c||c|c|c||c||c|c|c|}
\hline No. & Func. & Input & Prob. & No. & Func. & Input & Prob. \\
\hline \hline 1 & $f_{0}$ & $x_{1}$ & $1 / 2$ & 17 & $f_{1}$ & $x_{2}, x_{3}$ & $3 / 8$ \\
\hline 2 & $f_{0}$ & $x_{2}$ & $1 / 2$ & 18 & $f_{1}$ & $x_{2}, x_{5}$ & $3 / 8$ \\
\hline 3 & $f_{0}$ & $x_{3}$ & $3 / 4$ & 19 & $f_{1}$ & $x_{1}, x_{4}$ & $3 / 8$ \\
\hline 4 & $f_{0}$ & $x_{4}$ & $3 / 4$ & 20 & $f_{1}$ & $x_{1}, x_{2}$ & $3 / 8$ \\
\hline 5 & $f_{0}$ & $x_{5}$ & 0 & 21 & $f_{1}$ & $x_{1}, x_{3}$ & $5 / 8$ \\
\hline 6 & $f_{0}$ & $x_{1}, x_{2}$ & $1 / 2$ & 22 & $f_{1}$ & $x_{2}, x_{4}$ & $3 / 8$ \\
\hline 7 & $f_{0}$ & $x_{1}, x_{4}$ & $1 / 4$ & 23 & $f_{1}$ & $x_{3}, x_{5}$ & $3 / 8$ \\
\hline 8 & $f_{0}$ & $x_{3}, x_{5}$ & $1 / 4$ & 24 & $f_{1}$ & $x_{4}, x_{5}$ & $5 / 8$ \\
\hline 9 & $f_{0}$ & $x_{1}, x_{5}$ & $1 / 2$ & 25 & $f_{1}$ & $x_{3}, x_{4}$ & $3 / 8$ \\
\hline 10 & $f_{0}$ & $x_{3}, x_{4}$ & $3 / 4$ & 26 & $f_{1}$ & $x_{1}, x_{5}$ & $3 / 8$ \\
\hline 11 & $f_{0}$ & $x_{4}, x_{5}$ & $1 / 4$ & 27 & $f_{1}$ & $x_{2}, x_{3}, x_{4}$ & $5 / 8$ \\
\hline 12 & $f_{1}$ & $x_{1}$ & $5 / 8$ & 28 & $f_{2}$ & $x_{1}$ & $1 / 2$ \\
\hline 13 & $f_{1}$ & $x_{2}$ & 0 & 29 & $f_{2}$ & $x_{2}$ & $1 / 2$ \\
\hline 14 & $f_{1}$ & $x_{3}$ & $5 / 8$ & 30 & $f_{2}$ & $x_{3}$ & $1 / 2$ \\
\hline 15 & $f_{1}$ & $x_{4}$ & $5 / 8$ & 31 & $f_{2}$ & $x_{4}$ & $1 / 2$ \\
\hline 16 & $f_{1}$ & $x_{5}$ & $5 / 8$ & 32 & $f_{2}$ & $x_{5}$ & $1 / 2$ \\
\hline
\end{tabular}

\subsection{The Analysis of Input and Output Difference for Step Operation}

At each step, such as equation (5), message word $(X)$ having a difference has influence on a updating chaining variable $\left(A_{i}\right)$. Therefore, we need to take the following facts into consideration for difference analysis. The step operation uses addition modulo $2^{32}$. If addition modulo $2^{32}$ is substituted by $\oplus$, we must consider a probability according to a carry. If $k=31$, with respect to most significant 
bit, addition modulo $2^{32}$ and $\oplus$ play the same role. If $k \neq 31$, a carry happens with probability $1 / 2$. So, the analysis of input and output difference of step operation is done by equations (8), (9), (10), (11). $\alpha$ and $\beta$ denote arbitrary words, and $p$ denotes the probability satisfying a equation.

$$
\begin{aligned}
& \text { case } 1: k=31 \\
& \begin{array}{ll}
\left(\left(\alpha \oplus 1^{<<k}\right)+\beta\right) \oplus(\alpha+\beta)=1^{<<k} & (p=1) \\
\left(\left(\alpha \oplus 1^{<<k}\right)+\left(\beta \oplus 1^{<<k}\right)\right) \oplus(\alpha+\beta)=0 & (p=1)
\end{array} \\
& \left(\left(\alpha \oplus 1^{<<k}\right)+\beta\right) \oplus(\alpha+\beta)=1^{<<k} \quad(p=1 / 2) \\
& \left(\left(\alpha \oplus 1^{<<k}\right)+\left(\beta \oplus 1^{<<k}\right)\right) \oplus(\alpha+\beta)=0 \quad(p=1 / 2)
\end{aligned}
$$

\subsection{The Analysis of Expansion of Message Words}

By (1), (12) (19) are obtained as follows.

$$
\begin{aligned}
& X_{16}=\left(X_{0} \oplus X_{2} \oplus X_{7} \oplus X_{12}\right)^{<<1} \\
& X_{17}=\left(X_{1} \oplus X_{3} \oplus X_{8} \oplus X_{13}\right)^{<<1} \\
& X_{18}=\left(X_{2} \oplus X_{4} \oplus X_{9} \oplus X_{14}\right)^{<<1} \\
& X_{19}=\left(X_{3} \oplus X_{5} \oplus X_{10} \oplus X_{15}\right)^{<<1} \\
& X_{20}=\left(X_{4} \oplus X_{6} \oplus X_{11} \oplus X_{16}\right)^{<<1} \\
& X_{21}=\left(X_{5} \oplus X_{7} \oplus X_{12} \oplus X_{17}\right)^{<<1} \\
& X_{22}=\left(X_{6} \oplus X_{8} \oplus X_{13} \oplus X_{18}\right)^{<<1} \\
& X_{23}=\left(X_{7} \oplus X_{9} \oplus X_{14} \oplus X_{19}\right)^{<<1}
\end{aligned}
$$

Through the expansion of message words, message words which are affected by each $X_{i}(0 \leqslant i \leqslant 15)$ are shown on Table 4 below. This fact was shown by Han et al. at FSE 2002. When we take a look at the table 4, if we give a difference to $X_{0}, X_{16}$ and $X_{20}$ also have some differences.

Table 4. The effect of expansion of message words

\begin{tabular}{|l|l|l|l|l|l|l|l|l|l|l|l|l|l|l|l|}
\hline$X_{0}$ & $X_{1}$ & $X_{2}$ & $X_{3}$ & $X_{4}$ & $X_{5}$ & $X_{6}$ & $X_{7}$ & $X_{8}$ & $X_{9}$ & $X_{10}$ & $X_{11}$ & $X_{12}$ & $X_{13}$ & $X_{14}$ & $X_{15}$ \\
\hline \hline$X_{16}$ & $X_{17}$ & $X_{16}$ & $X_{17}$ & $X_{18}$ & $X_{19}$ & $X_{20}$ & $X_{16}$ & $X_{17}$ & $X_{18}$ & $X_{19}$ & $X_{20}$ & $X_{16}$ & $X_{17}$ & $X_{18}$ & $X_{19}$ \\
$X_{20}$ & $X_{21}$ & $X_{18}$ & $X_{19}$ & $X_{20}$ & $X_{21}$ & $X_{22}$ & $X_{20}$ & $X_{21}$ & $X_{22}$ & $X_{23}$ & & $X_{20}$ & $X_{21}$ & $X_{22}$ & $X_{23}$ \\
& & $X_{20}$ & $X_{21}$ & $X_{22}$ & $X_{23}$ & & $X_{21}$ & $X_{22}$ & $X_{23}$ & & & $X_{21}$ & $X_{22}$ & $X_{23}$ & \\
& & $X_{22}$ & $X_{23}$ & & & & $X_{23}$ & & & & & & & & \\
\hline
\end{tabular}

\subsection{The Analysis of Weakness of Shift Value Dependent on Message Words}

Generally, in case of MDx-hash functions, shift values are fixed. If shift values can be selected arbitrarily by attacker, a hash function using these shift values can be attacked easily by our attack method (described in Appendix A), regardless 
of the expansion of message words and ordering of message words and boolean functions and step operation. The original version of the hash function proposed at PKC'98 uses shift values dependent on message words. This principle of the design makes an attacker select shift values. So, in section 5 , based on this fact, we will attack the original version of the hash function proposed at PKC'98.

\section{The Selection of Message Blocks and Shift Values for Maximizing an Attack Probability}

\subsection{The Selection of Message Block Pair}

When we take a look at the table 4 , for the expansion of message words, it is impossible to give a difference to only one message word pair. But we can give differences to two message word pairs. Four cases exist.

$\left(X_{8}, X_{13}\right),\left(X_{9}, X_{14}\right),\left(X_{10}, X_{15}\right),\left(X_{11}, X_{20}\right)$. Out of four cases, we select $\left(X_{11}, X_{20}\right)$ for maximizing the probability of attack, because $\left(X_{11}, X_{20}\right)$ makes the smallest sum of four cases-the sums of the differences of steps of inputting $\left(X_{8}, X_{13}\right),\left(X_{9}, X_{14}\right),\left(X_{10}, X_{15}\right),\left(X_{11}, X_{20}\right)$ per round. The table of four cases is as follows.

Table 5. The differences of steps of inputting each word pair per round

\begin{tabular}{|c||c|c|c|c|}
\hline Round & $\left(X_{8}, X_{13}\right)$ & $\left(X_{9}, X_{14}\right)$ & $\left(X_{10}, X_{15}\right)$ & $\left(X_{11}, X_{20}\right)$ \\
\hline \hline 1 Round & 5 & 5 & 5 & 9 \\
\hline 2 Round & 13 & 1 & 11 & 4 \\
\hline 3 Round & 3 & 13 & 13 & 3 \\
\hline 4 Round & 20 & 18 & 4 & 7 \\
\hline \hline Sum & 41 & 37 & 33 & 23 \\
\hline
\end{tabular}

\subsection{The Selection of Differences of Message Block Pair and Shift Values}

The first standard giving differences to block pair is to give a difference to most significant bit for changing a modular addition with XOR operation. The second is to apply the same shift values to message block pair.

We select message block pair and shift values as follows.

$$
\begin{gathered}
\cdot X=\left(X_{i}\right)_{0 \leq i \leq 15}, \widetilde{X}=\left(\widetilde{X_{i}}\right)_{0 \leq i \leq 15}: \text { message block pair } \\
\cdot X_{11} \oplus X_{11}=1<<30\left(\widetilde{\left.X_{20} \oplus 1<<31=X_{20}\right)}\right. \\
\cdot \widetilde{X}_{i}=X_{i}(i \neq 11,20) \\
\cdot \text { Value of } 5 \text { low-order bits of message words } \\
l s b_{5}\left(X_{1}, X_{4}, X_{7}, X_{12}, X_{15}, X_{16}, X_{17}, X_{20}, X_{23}\right)=(10,0,21,21,11,0,10 \\
0,13)
\end{gathered}
$$




\subsection{Input Order of Message Words and Shift Values per Round through the Table}

Table 6 shows input order of message words and shift values per step.(M: order of message words, · : non-fixed value)

Table 6. The message input order and shift values per step

\begin{tabular}{|c|c|c|c|c|c|c|c|c|c|c|c|}
\hline step & $\mathrm{M}$ & shif & step & $\mathrm{M}$ & shift & step & $\mathrm{M}$ & shift & step & $\mathrm{M}$ & Sh1 \\
\hline$\overline{0}$ & 0 & 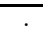 & 24 & 4 & $\overline{13}$ & 48 & 23 & $\overline{0}$ & $\overline{72}$ & 13 & \\
\hline 1 & 1 & . & 25 & 21 & . & 49 & 14 & . & 73 & 22 & 10 \\
\hline$\overline{2}$ & 2 & . & 26 & 17 & . & 50 & 19 & 10 & 74 & 2 & \\
\hline 3 & 3 & . & 27 & 1 & . & 51 & 21 & 10 & 75 & 14 & \\
\hline 4 & 4 & . & 28 & 23 & . & 52 & 13 & 13 & 76 & 3 & $\overline{0}$ \\
\hline 5 & 5 & . & 29 & 18 & 11 & 53 & 15 & 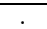 & 77 & 6 & . \\
\hline 6 & 6 & 21 & 30 & 12 & $\overline{0}$ & 54 & 20 & 21 & 78 & \begin{tabular}{|l|}
7 \\
\end{tabular} & \\
\hline 7 & \begin{tabular}{|l|}
7 \\
\end{tabular} & . & 31 & 10 & . & 55 & 8 & . & 79 & 5 & 21 \\
\hline 8 & \begin{tabular}{|l|}
8 \\
\end{tabular} & 11 & 32 & 5 & . & 56 & 18 & . & 80 & 15 & . \\
\hline 9 & \begin{tabular}{|l|}
9 \\
\end{tabular} & . & 33 & 16 & . & 57 & 11 & 0 & 81 & 0 & . \\
\hline 10 & 10 & . & 34 & 8 & . & 58 & 5 & . & 82 & 18 & . \\
\hline 11 & 11 & 13 & 35 & 0 & 0 & 59 & 4 & . & 83 & 23 & \\
\hline 12 & 12 & . & 36 & 20 & 21 & 60 & \begin{tabular}{|l|}
7 \\
\end{tabular} & $\overline{0}$ & 84 & 10 & 21 \\
\hline 13 & 13 & . & 37 & 3 & 10 & 61 & \begin{tabular}{|l|}
1 \\
\end{tabular} & . & 85 & 21 & . \\
\hline 14 & 14 & 0 & 38 & 22 & . & 62 & 9 & . & 86 & 16 & \\
\hline 15 & 15 & 0 & 39 & 6 & 21 & 63 & 12 & . & 87 & 20 & 11 \\
\hline 16 & 16 & 0 & 40 & 11 & . & 64 & 0 & . & 88 & 4 & $\overline{0}$ \\
\hline 17 & \begin{tabular}{|l|}
17 \\
\end{tabular} & 10 & 41 & 19 & . & 65 & \begin{tabular}{|l|}
2 \\
\end{tabular} & . & 89 & \begin{tabular}{|l|}
17 \\
\end{tabular} & 10 \\
\hline 18 & 18 & 21 & 42 & 15 & . & 66 & 6 & 11 & 90 & 12 & . \\
\hline 19 & 19 & 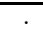 & 43 & 2 & 10 & 67 & 17 & . & 91 & 19 & \\
\hline 20 & 20 & . & 44 & 7 & r. & 68 & 10 & 21 & 92 & 8 & $\overline{0}$ \\
\hline 21 & 21 & $\cdot$ & 45 & 14 & & 69 & 22 & . & 93 & \begin{tabular}{|l|}
9 \\
\end{tabular} & . \\
\hline 22 & 22 & . & 46 & \begin{tabular}{|l|}
9 \\
\end{tabular} & 0 & 70 & 16 & . & 94 & 11 & \\
\hline 23 & 23 & 10 & 47 & 13 & & 71 & 3 & & 95 & 1 & 13 \\
\hline
\end{tabular}

\section{$5 \quad$ Full-Round Differential Attack}

From now on, we start to attack the original version of the hash function proposed at PKC'98 based on sections 3 and 4. Table 7 shows the full-round differential attack. Concretely we analyze steps generating differences. See Appendix A. $\triangle A_{i}, \triangle B_{i}, \triangle C_{i}, \triangle D_{i}, \triangle E_{i}$ mean the differences of chaining variables after a equation (5) and before a equation (6) at $i$ step.

The total probability is about $2^{-37.13}$. Therefore we can find a collision pair of the original version of the hash function proposed at PKC'98 with probability $2^{-37.13}$.

\section{Finding a Collision Pair in Practice by Simulation}

For finding a collision pair, we executed a program written in visual C 6.0 and running on a set of 10 PCs under Windows. From this, we found a collision pair in one computer with about 10 hours. The collision pair is as follows. 
Table 7. The Full-Round Differential Attack

\begin{tabular}{|c|c|c|c|c|c|c|c|c|c|c|c|c|c|c|c|}
\hline Step & $\triangle A$ & $\triangle B$ & $\triangle C$ & $\triangle D$ & $\Delta E$ & $\triangle X$ & $p$ & IStep & $\triangle A$ & $\triangle B$ & $\triangle C$ & $\triangle D$ & $\triangle E$ & $\Delta X$ & $p$ \\
\hline 0 & 0 & 0 & 0 & 0 & 0 & 0 & 1 & 48 & 0 & 0 & 0 & 0 & 0 & 0 & 1 \\
\hline 1 & 0 & 0 & 0 & 0 & 0 & 0 & 1 & 49 & 0 & 0 & 0 & 0 & 0 & 0 & 1 \\
\hline 2 & 0 & 0 & 0 & 0 & 0 & 0 & 1 & 50 & 0 & 0 & 0 & 0 & 0 & 0 & 1 \\
\hline 3 & 0 & 0 & \begin{tabular}{l|l}
0 \\
\end{tabular} & 0 & 0 & 0 & 1 & 51 & 0 & 0 & 0 & 0 & 0 & 0 & 1 \\
\hline 4 & 0 & 0 & 0 & 0 & 0 & 0 & 1 & 52 & 0 & 0 & 0 & 0 & 0 & 0 & 1 \\
\hline 5 & 0 & 0 & 0 & 0 & 0 & 0 & 1 & 53 & 0 & 0 & 0 & 0 & 0 & 0 & 1 \\
\hline 6 & 0 & 0 & 0 & 0 & 0 & 0 & 1 & 54 & 0 & $1<20$ & 0 & 0 & 0 & $1<31$ & 1 \\
\hline 7 & 0 & 0 & 0 & 0 & 0 & 0 & 1 & 55 & 0 & $1<30$ & 0 & 0 & 0 & 0 & $1 / 2$ \\
\hline 8 & 0 & 0 & \begin{tabular}{l|l}
0 \\
\end{tabular} & 0 & 0 & 0 & 1 & 56 & 0 & $1<30$ & 0 & 0 & 0 & 0 & $1 / 2$ \\
\hline 9 & 0 & 0 & 0 & 0 & 0 & 0 & 1 & 57 & 0 & $1<30$ & 0 & 0 & 0 & \begin{tabular}{|l|}
$<<30$ \\
\end{tabular} & $1 / 4$ \\
\hline 10 & 0 & 0 & 0 & 0 & 0 & 0 & 1 & 58 & 0 & $1<30$ & 0 & 0 & 0 & 0 & $1 / 2$ \\
\hline 11 & 0 & 0 & 0 & 0 & $1<11$ & $1<30$ & $1 / 2$ & 59 & 0 & 0 & 0 & 0 & 0 & 0 & $1 / 2$ \\
\hline 12 & 0 & 0 & 0 & 0 & $1<21$ & 0 & $1 / 2$ & 60 & 0 & 0 & 0 & 0 & 0 & 0 & 1 \\
\hline 13 & 0 & 0 & 0 & 0 & $1<21$ & 0 & $3 / 4$ & 61 & 0 & 0 & 0 & 0 & 0 & 0 & 1 \\
\hline 14 & 0 & 0 & 0 & 0 & $1<21$ & 0 & $3 / 4$ & 62 & 0 & 0 & 0 & 0 & $\overline{0}$ & 0 & 1 \\
\hline 15 & $1<21$ & 0 & 0 & 0 & $1<21$ & 0 & $1 / 2$ & 63 & 0 & 0 & 0 & 0 & 0 & 0 & 1 \\
\hline 16 & ${ }_{1}<31$ & 0 & 0 & 0 & $1<21$ & 0 & $1 / 4$ & 64 & 0 & 0 & 0 & 0 & 0 & 0 & 1 \\
\hline 17 & $1<31$ & 0 & 0 & 0 & $1<31$ & 0 & $3 / 8$ & 65 & 0 & $\overline{0}$ & 0 & 0 & 0 & 0 & 1 \\
\hline 18 & $1<31$ & 0 & 0 & 0 & $1<31$ & 0 & $3 / 4$ & 66 & 0 & 0 & 0 & 0 & 0 & 0 & 1 \\
\hline 19 & $1<31$ & 0 & 0 & 0 & $1<31$ & 0 & $1 / 4$ & 67 & 0 & 0 & 0 & 0 & 0 & 0 & 1 \\
\hline 20 & 0 & 0 & 0 & 0 & $1<31$ & $1<31$ & $1 / 2$ & 68 & 0 & 0 & 0 & 0 & 0 & 0 & 1 \\
\hline 21 & 0 & 0 & 0 & 0 & 0 & 0 & $1 / 2$ & 69 & 0 & 0 & 0 & 0 & 0 & 0 & 1 \\
\hline 22 & 0 & 0 & 0 & 0 & 0 & 0 & 1 & 70 & 0 & 0 & 0 & 0 & 0 & 0 & 1 \\
\hline 23 & 0 & 0 & 0 & 0 & 0 & 0 & 1 & 71 & 0 & 0 & 0 & 0 & 0 & 0 & 1 \\
\hline 24 & 0 & 0 & 0 & 0 & 0 & 0 & 1 & 72 & 0 & 0 & 0 & 0 & 0 & 0 & 1 \\
\hline 25 & 0 & 0 & 0 & 0 & 0 & 0 & 1 & 73 & 0 & 0 & 0 & 0 & 0 & 0 & 1 \\
\hline 26 & 0 & 0 & 0 & 0 & 0 & 0 & 1 & 74 & 0 & 0 & 0 & 0 & 0 & 0 & 1 \\
\hline 27 & 0 & 0 & 0 & 0 & 0 & 0 & 1 & 75 & 0 & 0 & 0 & 0 & 0 & 0 & 1 \\
\hline 28 & 0 & 0 & 0 & 0 & 0 & 0 & 1 & 76 & 0 & 0 & 0 & 0 & 0 & 0 & 1 \\
\hline 29 & 0 & 0 & 0 & 0 & 0 & 0 & 1 & 77 & 0 & 0 & 0 & 0 & 0 & 0 & 1 \\
\hline 30 & 0 & 0 & 0 & 0 & 0 & 0 & 1 & 78 & 0 & 0 & 0 & 0 & 0 & 0 & 1 \\
\hline 31 & 0 & 0 & 0 & 0 & 0 & 0 & 1 & 79 & 0 & 0 & 0 & 0 & 0 & 0 & 1 \\
\hline 32 & 0 & 0 & 0 & 0 & 0 & 0 & 1 & 80 & 0 & 0 & 0 & 0 & 0 & 0 & 1 \\
\hline 33 & 0 & 0 & 0 & 0 & 0 & 0 & 1 & 81 & 0 & 0 & 0 & 0 & 0 & 0 & 1 \\
\hline 34 & 0 & 0 & 0 & 0 & 0 & 0 & 1 & 82 & 0 & 0 & 0 & 0 & 0 & 0 & 1 \\
\hline 35 & 0 & 0 & 0 & 0 & 0 & 0 & 1 & 83 & 0 & 0 & 0 & 0 & 0 & 0 & 1 \\
\hline 36 & 0 & 0 & 0 & 0 & $1<20$ & $\mid 1<31$ & 1 & 84 & 0 & 0 & 0 & 0 & 0 & 0 & 1 \\
\hline 37 & 0 & 0 & 0 & $1<30$ & $1<30$ & 0 & $1 / 2$ & 85 & 0 & 0 & 0 & 0 & 0 & 0 & 1 \\
\hline 38 & 0 & 0 & 0 & $1<8$ & $1<30$ & 0 & $3 / 8$ & 86 & 0 & 0 & 0 & 0 & 0 & 0 & 1 \\
\hline 39 & 0 & 0 & 0 & $1<8$ & ${ }_{1}<30$ & 0 & $25 / 64$ & 87 & 0 & 0 & 0 & $1<10$ & 0 & $1<31$ & 1 \\
\hline 40 & 0 & 0 & 0 & ${ }_{1}<8$ & ${ }_{1}<30$ & $1<30$ & $15 / 128$ & 88 & 0 & 0 & $1<10$ & ${ }_{1}<20$ & 0 & 0 & $1 / 2$ \\
\hline 41 & 0 & 0 & 0 & $1<8$ & 0 & 0 & $25 / 64$ & 89 & 0 & $1<20$ & $1<20$ & $1<20$ & 0 & 0 & $5 / 16$ \\
\hline 42 & 0 & 0 & 0 & 0 & 0 & 0 & $5 / 8$ & 90 & 0 & $1<30$ & $1<20$ & $1<20$ & 0 & 0 & $5 / 8$ \\
\hline 43 & 0 & 0 & 0 & 0 & 0 & 0 & 1 & 91 & 0 & $1<30$ & $1<20$ & $1<20$ & 0 & 0 & $25 / 64$ \\
\hline 44 & 0 & 0 & 0 & 0 & 0 & 0 & 1 & 92 & 0 & $1<30$ & $1<20$ & 0 & 0 & 0 & $15 / 64$ \\
\hline 45 & 0 & 0 & 0 & 0 & 0 & 0 & 1 & 93 & 0 & $1<30$ & 0 & 0 & 0 & 0 & $25 / 64$ \\
\hline 46 & 0 & 0 & 0 & 0 & 0 & 0 & 1 & 94 & 0 & 0 & 0 & 0 & 0 & $1<30$ & $3 / 16$ \\
\hline 47 & 0 & 0 & 0 & 0 & 0 & 0 & 1 & 95 & 0 & 0 & 0 & 0 & 0 & 0 & 1 \\
\hline
\end{tabular}




\begin{tabular}{|c|c|c|c|}
\hline$X_{0}=0 x d f 407 f 1 a$ & $X_{1}=0 \times 99 \mathrm{c} 0464 \mathrm{a}$ & $X_{2}=0 \times 3380 \mathrm{a} 1 \mathrm{fa}$ & $X_{3}=0 \mathrm{x} 0 \mathrm{~d} 40 \mathrm{be} 50$ \\
\hline$X_{4}=0 \mathrm{x} 6580 \mathrm{c} 1 \mathrm{c} 0$ & $X_{5}=0 \times \mathrm{xb} 8803020$ & $X_{6}=0 x f 5 c 09 a 9 e$ & $X_{7}=0$ \\
\hline$X_{8}=0 \mathrm{x} 1 \mathrm{f} 005106$ & $X_{9}=0 \mathrm{x}$ & $X_{10}=$ & $X_{11}=0$ \\
\hline$-0 \times$ & $X_{13}=0$ & $X_{14}=0 \times 28 c 04748$ & $X_{15}=0 \mathrm{x}$ \\
\hline & \multicolumn{3}{|c|}{$\widetilde{X_{11}}=X_{11} \oplus 1^{<<30}, \widetilde{X_{i}}=X_{i}(i \neq 11)$} \\
\hline
\end{tabular}

The output of compression function for two message blocks is as follows.

\section{Oxfe684dca $\quad$ 0x33524aa4 $\quad$ 0x15ce9f59 $0 x d 200 e 689$ 0x7b01f656}

And a collision pair on the compression function leads to a collision on the full hash function by simply appending identical blocks or the padding fields for same-length messages.

\section{On the Security of Reduced Versions of 3-Pass HAVAL}

HAVAL is a dedicated hash function of the MD family which was proposed by Zheng et al[11]. Kasselman et al. found collisions for the last two passes of 3-pass HAVAL 6]. Park et al., at ACISP 2002, found a 256-bit collision of the first two passes of 3-pass HAVAL and of the last two passes of 3-pass HAVAL 7 . In this paper, we attack reduced versions of 3-pass HAVAL using our method different from two previous attack methods(1-2 round: attack probability is $2^{-18}, 2-3$ round: attack probability is $\left.2^{-50}\right)$. Concretely, we select two messages as follow. For 1-2 round of HAVAL, $W_{29} \oplus \widetilde{W_{29}}=1^{<<31}, \widetilde{W_{31}} \oplus 1^{<<20}=W_{31}$. For $2-3$ round of HAVAL, $W_{7} \oplus \widetilde{W_{7}}=1^{<<31}, \widetilde{W_{31}} \oplus 1^{<<30}=W_{31}$. (See Appendix B: Table 8, 9)

\section{Conclusion}

This paper shows that we can find a collision pair from the original hash function with probability $2^{-37.13}$ not transforming boolean functions. This means that a weakness of the hash function proposed at PKC'98 comes from the shift values of the hash function. That is, shift values depending on messages can be a factor of reducing the security of hash functions. Also, our attack method is applied to the reduced version of HAVAL because HAVAL has the weakness of message input order.

Therefore shift values have to be carefully chosen for the security of hash functions. And in case that shift values are fixed, the message input order have to be carefully chosen, also.

\section{Acknowledgements}

We thank to the anonymous referees for their useful comments. 


\section{References}

1. F. Chabaud and A. Joux, Differential Collisions in SHA-0, Advances in CRYPTO'98, LNCS 1462, Springer-Verlag, 1998, pp. 56-71.

2. H. Dobbertin, Cryptanalysis of MD4, Fast Software Encryption, LNCS 1039, Springer-Verlag, 1996, pp. 53-69.

3. H. Dobbertin, Cryptanalysis of MD5 Compress, May. 1996. www-cse.ucsd.edu/users/bsy/dobbertin.ps

4. Sanguk Shin, Kyunghyune Rhee, Daehyun Ryu, Sangjin Lee, A New Hash Function Based on MDx-family and Its Application to MAC, Public Key Cryptography'98, pp. 234-246. 1998.

5. Daewan Han, Sangwoo Park, Seongtaek Chee, Cryptanalysis of a Hash Function Proposed at PKC'98, Fast Software Encryption 2002, LNCS 2365, pp. 252-262.

6. P.R. Kasselman and W.T. Penzhorn, Cryptanalysis of reduced version of HAVAL, Electronics Letters 6th January 2000 Vol.36 No.1, pp. 30-31.

7. S.W. Park, S.H. Sung, S.T. Chee, J.I. Lim, On the Security of Reduced Versions of 3-Pass HAVAL, ACISP 2002, LNCS 2384, pp. 406-419.

8. R. Rivest, The MD4 message digest algorithm, RFC 1320, Internet Activities Board, Internet Privacy Task Force, Apr. 1992.

9. R. Rivest, The MD5 message digest algorithm, RFC 1321, Internet Activities Board, Internet Privacy Task Force, Apr. 1992.

10. Federal Information Processing Standards Publication 180-1, April 17, 1995.

11. Y. Zheng, J. Pieprzyk and J. Sebberry, HAVAL - A one-way hashing algorithm with variable length of output, Advances in Cryptology-Auscrypt'92, LNCS 718, SpringerVerlag, 1993, pp. 83-104.

\section{Appendix A}

\section{The Analysis of $11 \sim 21$ Steps of Table 7}

The first round use the function $f_{0}$. The values of Table 7 are calculated as follows.

$$
\begin{gathered}
\triangle E_{11}=\left[f_{0}\left(E_{10}, A_{10}, B_{10}, C_{10}, D_{10}\right)+X_{11}+K_{1}\right]^{<<S_{11}=13} \oplus\left[f _ { 0 } \left(E_{10}, A_{10}, B_{10}, C_{10},\right.\right. \\
\left.\left.D_{10}\right)+\left(X_{11} \oplus 1^{<<30}\right)+K_{1}\right]^{<<S_{11}=13}=1^{<<11} \quad(p=1 / 2)
\end{gathered}
$$

Above equality holds with probability $1 / 2$ by $(10)$.

$$
\begin{array}{cc}
\triangle D_{12}=\left[f_{0}\left(D_{11}, E_{11}, A_{11}, B_{11}, C_{11}\right)+X_{12}+K_{1}\right]^{<<S_{12}} \oplus\left[f _ { 0 } \left(D_{11}, E_{11} \oplus 1^{<<11}, A_{11},\right.\right. \\
\left.\left.B_{11}, C_{11}\right)+X_{12}+K_{1}\right]^{<<S_{12}}=0 & (p=1 / 2)
\end{array}
$$

Above equality holds with probability $1 / 2$ by 2 of Table 3 .

$$
\begin{array}{r}
\triangle C_{13}=\left[f_{0}\left(C_{12}, D_{12}, E_{12}, A_{12}, B_{12}\right)+X_{13}+K_{1}\right]^{<<S_{13}} \oplus\left[f _ { 0 } \left(C_{12}, D_{12}, E_{12} \oplus\right.\right. \\
1^{<<21}, A_{12}, \\
\left.\left.B_{12}\right)+X_{13}+K_{1}\right]^{<<S_{13}}=0
\end{array}
$$

Above equality holds with probability $3 / 4$ by 3 of Table 3 .

$$
\begin{array}{cc}
\triangle B_{14}= & {\left[f_{0}\left(B_{13}, C_{13}, D_{13}, E_{13}, A_{13}\right)+X_{14}+K_{1}\right]^{<<S_{14}=0} \oplus\left[f _ { 0 } \left(B_{13}, C_{13}, D_{13}, E_{13}\right.\right.} \\
\left.\left.\oplus 1^{<<21}, A_{13}\right)+X_{14}+K_{1}\right]^{<<S_{14}=0}=0 & (p=3 / 4)
\end{array}
$$

Above equality holds with probability $3 / 4$ by 4 of Table 3 . 


$$
\begin{array}{rr}
\triangle A_{15}=\left[f_{0}\left(A_{14}, B_{14}, C_{14}, D_{14}, E_{14}\right)+X_{15}+K_{1}\right]^{<<S_{15}=0} \oplus\left[f _ { 0 } \left(A_{14}, B_{14}, C_{14}, D_{14},\right.\right. \\
\left.\left.E_{14} \oplus 1^{<<21}\right)+X_{15}+K_{1}\right]^{<<S_{15}=0}=1^{<<21} & (p=1 / 2)
\end{array}
$$

Above equality holds with probability $1 / 2$ by (10) and by 5 of Table 3 .

$$
\begin{array}{r}
\triangle E_{16}=\left[f_{0}\left(E_{15}, A_{15}, B_{15}, C_{15}, D_{15}\right)+X_{16}+K_{1}\right]^{<<S_{16}=0} \oplus\left[f _ { 0 } \left(E_{15} \oplus 1^{<<21}, A_{15} \oplus\right.\right. \\
\left.\left.1^{<<21}, B_{15}, C_{15}, D_{15}\right)+X_{16}+K_{1}\right]^{<<S_{16}=0}=1^{<<21} \quad(p=1 / 4)
\end{array}
$$

Above equality holds with probability $1 / 4$ by (10) and by 6 of Table 3 .

$$
\begin{array}{rr}
\triangle D_{17} & =\left[f_{0}\left(D_{16}, E_{16}, A_{16}, B_{16}, C_{16}\right)+X_{17}+K_{1}\right]^{<<S_{17}=10} \oplus\left[f _ { 0 } \left(D_{16}, E_{16} \oplus\right.\right. \\
1^{<<21}, A_{16} & (p=3 / 8)
\end{array}
$$$$
(p=3 / 8)
$$

Above equality holds with probability $3 / 8$ by 2,3 of Table 3 .

$$
\begin{gathered}
\triangle C_{18}=\left[f_{0}\left(C_{17}, D_{17}, E_{17}, A_{17}, B_{17}\right)+X_{18}+K_{1}\right]^{<<S_{18}=21} \oplus\left[f _ { 0 } \left(C_{17}, D_{17}, E_{17} \oplus\right.\right. \\
\left.\left.1^{<<31}, A_{17} \oplus 1^{<<31}, B_{17}\right)+X_{18}+K_{1}\right]^{<<S_{18}=21}=0 \quad(p=3 / 4)
\end{gathered}
$$

Above equality holds with probability $3 / 4$ by 10 of Table 3 .

$$
\begin{array}{cr}
\triangle B_{19}=\left[f_{0}\left(B_{18}, C_{18}, D_{18}, E_{18}, A_{18}\right)+X_{19}+K_{1}\right]^{<<S_{19}} \oplus\left[f _ { 0 } \left(B_{18}, C_{18}, D_{18}, E_{18} \oplus\right.\right. \\
\left.\left.1^{<<31}, A_{18} \oplus 1^{<<31}\right)+X_{19}+K_{1}\right]^{<<S_{19}}=0 & (p=1 / 4)
\end{array}
$$

Above equality holds with probability $1 / 4$ by 11 of Table 3 .

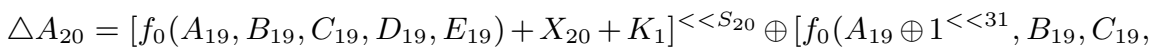

$$
\begin{aligned}
& \left.\left.D_{19}, E_{19} \oplus 1^{<<31}\right)+\left(X_{20} \oplus 1^{<<31}\right)+K_{1}\right]^{<<S_{20}}=0 \quad(p=1 / 2) \\
& \triangle E_{21}=\left[f_{0}\left(E_{20}, A_{20}, B_{20}, C_{20}, D_{20}\right)+X_{21}+K_{1}\right]^{<<S_{21}} \oplus\left[f _ { 0 } \left(E_{20} \oplus 1^{<<31}, A_{20}, B_{20},\right.\right. \\
& \left.\left.C_{20}, D_{20}\right)+X_{21}+K_{1}\right]^{<<S_{21}}=0 \quad(p=1 / 2)
\end{aligned}
$$

\section{The Analysis of $36 \sim 42$ Steps of Table 7}

The probabilities of following equalities also are calculated like the above method.

$$
\begin{aligned}
& \triangle E_{36}=\left[f_{1}\left(E_{35}, A_{35}, B_{35}, C_{35}, D_{35}\right)+X_{20}+K_{2}\right]^{<<S_{36}=21} \oplus\left[f _ { 1 } \left(E_{35}, A_{35}, B_{35}, C_{35},\right.\right. \\
& \left.\left.D_{35}\right)+\left(X_{20} \oplus 1^{<<31}\right)+K_{2}\right]^{<<S_{36}=21}=1^{<<20} \quad(p=1) \\
& \triangle D_{37}=\left[f_{1}\left(D_{36}, E_{36}, A_{36}, B_{36}, C_{36}\right)+X_{3}+K_{2}\right]^{<<S_{37}=10} \oplus\left[f _ { 1 } \left(D_{36}, E_{36} \oplus 1^{<<20}, A_{36},\right.\right. \\
& \left.\left.B_{36}, C_{36}\right)+X_{3}+K_{2}\right]^{<<S_{37}=10}=1^{<<30} \quad(p=1 / 2) \\
& \triangle C_{38}=\left[f_{1}\left(C_{37}, D_{37}, E_{37}, A_{37}, B_{37}\right)+X_{22}+K_{2}\right]^{<<S_{38}} \oplus\left[f _ { 1 } \left(C_{37}, D_{37} \oplus 1^{<<30}, E_{37}\right.\right. \\
& \left.\left.\oplus 1^{<<30}, A_{37}, B_{37}\right)+X_{22}+K_{2}\right]^{<<S_{38}}=0 \quad(p=3 / 8) \\
& \triangle B_{39}=\left[f_{1}\left(B_{38}, C_{38}, D_{38}, E_{38}, A_{38}\right)+X_{6}+K_{2}\right]^{<<S_{39}=21} \oplus\left[f _ { 1 } \left(B_{38}, C_{38}, D_{38}\right.\right. \\
& \left.\left.\oplus 1^{<<8}, E_{38} \oplus 1^{<<30}, A_{38}\right)+X_{6}+K_{2}\right]^{<<S_{39}=21}=0 \quad(p=25 / 64) \\
& \triangle A_{40}=\left[f_{1}\left(A_{39}, B_{39}, C_{39}, D_{39}, E_{39}\right)+X_{11}+K_{2}\right]^{<<S_{40}} \oplus\left[f _ { 1 } \left(A_{39}, B_{39}, C_{39}, D_{39}\right.\right. \\
& \left.\left.\oplus 1^{<<8}, E_{39} \oplus 1^{<<30}\right)+\left(X_{11} \oplus 1^{<<30}\right)+K_{2}\right]^{<<S_{40}}=0 \quad(p=15 / 128) \\
& \triangle E_{41}=\left[f_{1}\left(E_{40}, A_{40}, B_{40}, C_{40}, D_{40}\right)+X_{19}+K_{2}\right]^{<<S_{41}} \oplus\left[f _ { 1 } \left(E_{40} \oplus 1^{<<30}, A_{40}, B_{40},\right.\right. \\
& \left.\left.C_{40}, D_{40} \oplus 1^{<<8}\right)+X_{19}+K_{2}\right]^{<<S_{41}}=0 \quad(p=25 / 64) \\
& \triangle D_{42}=\left[f_{1}\left(D_{41}, E_{41}, A_{41}, B_{41}, C_{41}\right)+X_{15}+K_{2}\right]^{<<S_{42}} \oplus\left[f _ { 1 } \left(D_{41} \oplus 1^{<<8}, E_{41}, A_{41}\right.\right. \text {, } \\
& \left.\left.B_{41}, C_{41}\right)+X_{15}+K_{2}\right]^{<<S_{42}}=0 \\
& (p=5 / 8)
\end{aligned}
$$




\section{The Analysis of 54 59 Steps of Table 7}

The probabilities of following equalities also are calculated like the above method.

$$
\begin{array}{rr}
\triangle B_{54}= & {\left[f_{2}\left(B_{53}, C_{53}, D_{53}, E_{53}, A_{53}\right)+X_{20}+K_{3}\right]^{<<S_{54}=21} \oplus\left[f _ { 2 } \left(B_{53}, C_{53}, D_{53}, E_{53},\right.\right.} \\
\left.\left.A_{53}\right)+\left(X_{20} \oplus 1^{<<31}\right)+K_{3}\right]^{<<S_{54}=21}=1^{<<20} & (p=1)
\end{array}
$$

$\triangle A_{55}=\left[f_{2}\left(A_{54}, B_{54}, C_{54}, D_{54}, E_{54}\right)+X_{8}+K_{3}\right]^{<<S_{55}} \oplus\left[f_{2}\left(A_{54}, B_{54} \oplus 1^{<<20}, C_{54}\right.\right.$,

$$
\left.\left.D_{54}, E_{54}\right)+X_{8}+K_{3}\right]^{<<S_{55}}=0 \quad(p=1 / 2)
$$

$\triangle E_{56}=\left[f_{2}\left(E_{55}, A_{55}, B_{55}, C_{55}, D_{55}\right)+X_{18}+K_{3}\right]^{<<S_{56}} \oplus\left[f_{2}\left(E_{55}, A_{55}, B_{55} \oplus 1^{<<30}\right.\right.$,

$$
\left.\left.C_{55}, D_{55}\right)+X_{18}+K_{3}\right]^{<<S_{56}}=0 \quad(p=1 / 2)
$$

$\triangle D_{57}=\left[f_{2}\left(D_{56}, E_{56}, A_{56}, B_{56}, C_{56}\right)+X_{11}+K_{3}\right]^{<<S_{57}=0} \oplus\left[f_{2}\left(D_{56}, E_{56}, A_{56}, B_{56}\right.\right.$

$$
\left.\left.\oplus 1^{<<30}, C_{56}\right)+\left(X_{11} \oplus 1^{<<30}\right)+K_{3}\right]^{<<S_{57}=0}=0 \quad(p=1 / 4)
$$

$\triangle C_{58}=\left[f_{2}\left(C_{57}, D_{57}, E_{57}, A_{57}, B_{57}\right)+X_{5}+K_{3}\right]^{<<S_{58}} \oplus\left[f_{2}\left(C_{57}, D_{57}, E_{57}, A_{57}, B_{57}\right.\right.$

$$
\begin{array}{cc}
\left.\left.\oplus 1^{<<30}\right)+X_{5}+K_{3}\right]^{<<S_{58}}=0 & (p=1 / 2) \\
\triangle B_{59}=\left[f_{2}\left(B_{58}, C_{58}, D_{58}, E_{58}, A_{58}\right)+X_{4}+K_{3}\right]^{<<S_{59}} \oplus\left[f _ { 2 } \left(B_{58} \oplus 1^{<<30}, C_{58}, D_{58},\right.\right.
\end{array}
$$

$$
\left.\left.E_{58}, A_{58}\right)+X_{4}+K_{3}\right]^{<<S_{59}}=0 \quad(p=1 / 2)
$$

\section{The Analysis of $87 \sim 95$ Steps of Table 7}

The probabilities of following equalities also are calculated like the above method.

$$
\begin{array}{rr}
\triangle D_{87}=\left[f_{1}\left(D_{86}, E_{86}, A_{86}, B_{86}, C_{86}\right)+X_{20}+K_{4}\right]^{<<S_{87}=11} \oplus\left[f _ { 1 } \left(D_{86}, E_{86}, A_{86}, B_{86},\right.\right. \\
\left.\left.C_{86}\right)+\left(X_{20} \oplus 1^{<<31}\right)+K_{4}\right]^{<<S_{87}=11}=1^{<<10}
\end{array}
$$

$\triangle C_{88}=\left[f_{1}\left(C_{87}, D_{87}, E_{87}, A_{87}, B_{87}\right)+X_{4}+K_{4}\right]^{<<S_{88}=0} \oplus\left[f_{1}\left(C_{87}, D_{87} \oplus 1^{<<10}, E_{87}\right.\right.$,

$$
\left.\left.A_{87}, B_{87}\right)+X_{4}+K_{4}\right]^{<<S_{88}=0}=1^{<<10} \quad(p=1 / 2)
$$

$\triangle B_{89}=\left[f_{1}\left(B_{88}, C_{88}, D_{88}, E_{88}, A_{88}\right)+X_{17}+K_{4}\right]^{<<S_{89}=10} \oplus\left[f_{1}\left(B_{88}, C_{88} \oplus 1^{<<10}, D_{88}\right.\right.$ $\left.\left.\oplus 1^{<<20}, E_{88}, A_{88}\right)+X_{17}+K_{4}\right]^{<<S_{89}=10}=1^{<<20} \quad(p=5 / 16)$ $\triangle A_{90}=\left[f_{1}\left(A_{89}, B_{89}, C_{89}, D_{89}, E_{89}\right)+X_{12}+K_{4}\right]^{<<S_{90}} \oplus\left[f_{1}\left(A_{89}, B_{89} \oplus 1^{<<20}, C_{89}\right.\right.$ $\left.\left.\oplus 1^{<<20}, D_{89} \oplus 1^{<<20}, E_{89}\right)+X_{12}+K_{4}\right]^{<<S_{90}}=0 \quad(p=5 / 8)$ $\triangle E_{91}=\left[f_{1}\left(E_{90}, A_{90}, B_{90}, C_{90}, D_{90}\right)+X_{19}+K_{4}\right]^{<<S_{91}} \oplus\left[f_{1}\left(E_{90}, A_{90}, B_{90} \oplus 1^{<<30}\right.\right.$,

$\left.\left.C_{90} \oplus 1^{<<20}, D_{90} \oplus 1^{<<20}\right)+X_{19}+K_{4}\right]^{<<S_{91}}=0 \quad(p=25 / 64)$ $\triangle D_{92}=\left[f_{1}\left(D_{91}, E_{91}, A_{91}, B_{91}, C_{91}\right)+X_{8}+K_{4}\right]^{<<S_{92}=0} \oplus\left[f_{1}\left(D_{91} \oplus 1^{<<20}, E_{91}, A_{91}\right.\right.$, $\left.\left.B_{91} \oplus 1^{<<30}, C_{91} \oplus 1^{<<20}\right)+X_{8}+K_{4}\right]^{<<S_{92}=0}=0 \quad(p=15 / 64)$ $\triangle C_{93}=\left[f_{1}\left(C_{92}, D_{92}, E_{92}, A_{92}, B_{92}\right)+X_{9}+K_{4}\right]^{<<S_{93}} \oplus\left[f_{1}\left(C_{92} \oplus 1^{<<20}, D_{92}, E_{92}, A_{92}\right.\right.$, $\left.\left.B_{92} \oplus 1^{<<30}\right)+X_{9}+K_{4}\right]^{<<S_{93}}=0 \quad(p=25 / 64)$

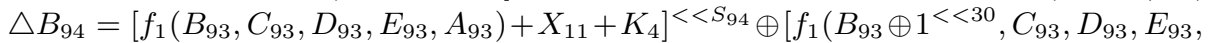
$\left.\left.A_{93}\right)+\left(X_{11} \oplus 1^{<<30}\right)+K_{4}\right]^{<<S_{94}}=0 \quad(p=3 / 16)$ $\triangle A_{95}=\left[f_{1}\left(A_{94}, B_{94}, C_{94}, D_{94}, E_{94}\right)+X_{1}+K_{4}\right]^{<<S_{95}=13} \oplus\left[f_{1}\left(A_{94}, B_{94}, C_{94}, D_{94}, E_{94}\right)\right.$ $\left.+X_{1}+K_{4}\right]^{<<S_{95}=13}=0$ 


\section{Appendix B}

Table 8. Attack on the first two passes of 3-pass HAVAL

\begin{tabular}{|c|c|c|c|c|c|c|c|c|c|c|c|c|c|c|c|c|c|c|c|c|c|}
\hline tep & $\triangle A$ & $\triangle B$ & $\triangle C$ & $\triangle D$ & $\triangle E$ & $\triangle F$ & $\triangle G$ & $\triangle H$ & $\triangle X$ & $p$ & Step & $\triangle A$ & $\triangle B$ & $\triangle C$ & $\triangle D$ & $\Delta E$ & $\triangle F$ & $\triangle G$ & $\triangle H$ & $\triangle X$ & $p$ \\
\hline 0 & 0 & 0 & 0 & 0 & 0 & 0 & 0 & 0 & 0 & 1 & 32 & 0 & 0 & 0 & 0 & 0 & 0 & 0 & 0 & 0 & 1 \\
\hline 1 & 0 & 0 & 0 & 0 & 0 & 0 & 0 & 0 & 0 & 1 & 33 & 0 & 0 & 0 & 0 & 0 & 0 & 0 & 0 & 0 & 1 \\
\hline 2 & 0 & 0 & 0 & 0 & 0 & 0 & 0 & 0 & 0 & 1 & 34 & 0 & 0 & 0 & 0 & 0 & 0 & 0 & 0 & 0 & 1 \\
\hline 3 & 0 & 0 & 0 & 0 & 0 & 0 & 0 & 0 & 0 & 1 & 35 & 0 & 0 & 0 & 0 & 0 & 0 & 0 & 0 & 0 & 1 \\
\hline 4 & 0 & 0 & 0 & 0 & 0 & 0 & 0 & 0 & 0 & 1 & 36 & 0 & 0 & 0 & 0 & 0 & 0 & 0 & 0 & 0 & 1 \\
\hline 5 & 0 & 0 & 0 & 0 & 0 & 0 & 0 & 0 & 0 & 1 & 37 & 0 & 0 & 0 & 0 & 0 & 0 & 0 & 0 & 0 & 1 \\
\hline 6 & 0 & 0 & 0 & 0 & 0 & 0 & 0 & 0 & 0 & 1 & 38 & 0 & $1<31$ & 0 & 0 & 0 & 0 & 0 & 0 & $1<31$ & 1 \\
\hline 7 & ${ }_{1}<31$ & 0 & 0 & 0 & 0 & 0 & 0 & 0 & $1<31$ & 1 & 39 & 0 & ${ }_{1}<31$ & 0 & 0 & 0 & 0 & 0 & 0 & 0 & $1 / 2$ \\
\hline 8 & $1<31$ & 0 & 0 & 0 & 0 & 0 & 0 & 0 & 0 & $1 / 2$ & 40 & 0 & $1<31$ & 0 & 0 & 0 & 0 & 0 & 0 & 0 & $1 / 2$ \\
\hline 9 & $1<31$ & 0 & 0 & 0 & 0 & 0 & 0 & 0 & 0 & $1 / 2$ & 41 & 0 & $1<31$ & 0 & 0 & 0 & 0 & $\overline{0}$ & 0 & 0 & $1 / 2$ \\
\hline 10 & $1<31$ & 0 & 0 & 0 & 0 & 0 & 0 & 0 & 0 & $1 / 2$ & 42 & 0 & $1<31$ & 0 & 0 & 0 & 0 & 0 & 0 & 0 & $1 / 2$ \\
\hline 11 & $1<31$ & 0 & 0 & 0 & 0 & 0 & 0 & 0 & 0 & $1 / 2$ & 43 & 0 & $1<31$ & 0 & 0 & 0 & 0 & 0 & 0 & 0 & $1 / 2$ \\
\hline 12 & $1<31$ & 0 & 0 & 0 & 0 & 0 & 0 & 0 & 0 & $1 / 2$ & 44 & 0 & $1<31$ & 0 & 0 & 0 & 0 & 0 & 0 & 0 & $1 / 2$ \\
\hline 13 & $1_{1<31}$ & 0 & 0 & 0 & 0 & 0 & 0 & 0 & 0 & $1 / 2$ & 45 & 0 & $1<31$ & \begin{tabular}{|l|}
0 \\
\end{tabular} & $\overline{0}$ & 0 & 0 & 0 & 0 & 0 & $1 / 2$ \\
\hline 14 & ${ }_{1}<31$ & 0 & 0 & 0 & 0 & 0 & 0 & 0 & 0 & $1 / 2$ & 46 & 0 & $1<20$ & 0 & 0 & 0 & 0 & 0 & 0 & 0 & $1 / 2$ \\
\hline 15 & $1<20$ & 0 & 0 & 0 & 0 & 0 & 0 & 0 & 0 & $1 / 2$ & 47 & 0 & $1<20$ & 0 & 0 & 0 & 0 & 0 & 0 & 0 & $1 / 2$ \\
\hline 16 & $1<20$ & 0 & 0 & 0 & 0 & 0 & 0 & 0 & 0 & $1 / 2$ & 48 & 0 & $1<20$ & 0 & 0 & 0 & 0 & 0 & 0 & 0 & $1 / 2$ \\
\hline 17 & $1<20$ & 0 & 0 & 0 & 0 & 0 & 0 & $\overline{0}$ & 0 & $1 / 2$ & 49 & 0 & $1<20$ & 0 & 0 & 0 & 0 & 0 & $\overline{0}$ & 0 & $1 / 2$ \\
\hline 18 & $1<20$ & 0 & 0 & 0 & 0 & 0 & 0 & 0 & 0 & $1 / 2$ & 50 & 0 & $1<20$ & 0 & 0 & 0 & 0 & 0 & 0 & 0 & $1 / 2$ \\
\hline 19 & $1<20$ & 0 & 0 & 0 & 0 & 0 & 0 & 0 & 0 & $1 / 2$ & 51 & 0 & $1<20$ & 0 & 0 & 0 & 0 & 0 & 0 & 0 & $1 / 2$ \\
\hline 20 & $1<20$ & 0 & 0 & 0 & 0 & 0 & 0 & 0 & 0 & $1 / 2$ & 52 & 0 & $1<20$ & 0 & 0 & $\overline{0}$ & 0 & 0 & 0 & 0 & $1 / 2$ \\
\hline 21 & $1<20$ & 0 & 0 & 0 & 0 & 0 & 0 & 0 & 0 & $1 / 2$ & 53 & 0 & $1<20$ & 0 & $\overline{0}$ & 0 & 0 & 0 & 0 & 0 & $1 / 2$ \\
\hline 22 & $1<20$ & 0 & 0 & 0 & 0 & 0 & 0 & 0 & 0 & $1 / 2$ & 54 & 0 & $1<9$ & 0 & 0 & 0 & 0 & 0 & 0 & 0 & $1 / 2$ \\
\hline 23 & $1<9$ & 0 & 0 & 0 & 0 & 0 & 0 & 0 & 0 & $1 / 2$ & 55 & 0 & $1<9$ & 0 & 0 & 0 & 0 & 0 & 0 & 0 & $1 / 2$ \\
\hline 24 & $1<9$ & 0 & 0 & 0 & 0 & 0 & 0 & 0 & 0 & $1 / 2$ & 56 & 0 & $1<9$ & 0 & 0 & 0 & 0 & 0 & $\overline{0}$ & 0 & $1 / 2$ \\
\hline 25 & $1<9$ & 0 & 0 & 0 & 0 & 0 & 0 & 0 & 0 & $1 / 2$ & 57 & 0 & $1<9$ & 0 & 0 & 0 & 0 & 0 & 0 & 0 & $1 / 2$ \\
\hline 26 & $1<9$ & 0 & 0 & 0 & 0 & 0 & 0 & 0 & 0 & $1 / 2$ & 58 & 0 & $1<9$ & 0 & 0 & 0 & 0 & 0 & 0 & 0 & $1 / 2$ \\
\hline 27 & $1<9$ & 0 & 0 & 0 & 0 & 0 & 0 & 0 & 0 & $1 / 2$ & 59 & 0 & $1<9$ & 0 & 0 & 0 & 0 & 0 & 0 & 0 & $1 / 2$ \\
\hline 28 & $1<9$ & 0 & 0 & \begin{tabular}{|l|}
0 \\
\end{tabular} & 0 & 0 & 0 & 0 & 0 & $1 / 2$ & 60 & 0 & $1<9$ & 0 & 0 & 0 & 0 & 0 & 0 & 0 & $1 / 2$ \\
\hline 29 & $1<9$ & 0 & 0 & 0 & 0 & 0 & 0 & 0 & 0 & $1 / 2$ & 61 & 0 & $1<9$ & 0 & 0 & 0 & 0 & 0 & 0 & 0 & $1 / 2$ \\
\hline 30 & $1<9$ & 0 & 0 & 0 & 0 & 0 & 0 & 0 & 0 & $1 / 2$ & 62 & 0 & 0 & 0 & 0 & 0 & 0 & 0 & 0 & $1<30$ & $1 / 4$ \\
\hline 31 & 0 & 0 & 0 & 0 & 0 & 0 & 0 & 0 & $1<30$ & $1 / 4$ & 63 & 0 & 0 & 0 & 0 & 0 & 0 & 0 & 0 & 0 & 1 \\
\hline
\end{tabular}

$\square$ : The updated part per step 
Table 9. Attack on the last two passes of 3-pass HAVAL

\begin{tabular}{|c|c|c|c|c|c|c|c|c|c|c|c|c|c|c|c|c|c|c|c|c|c|}
\hline Step & $\triangle A$ & $\triangle B$ & $\triangle C$ & $\triangle D$ & $\triangle E$ & $\triangle F$ & $\triangle G$ & $\triangle H$ & $\triangle X$ & $p$ & IStep & $\| \triangle A$ & $\triangle B$ & $\triangle C$ & $\triangle D$ & $\triangle E$ & $\triangle F$ & $\triangle G$ & $\triangle H$ & $\triangle X$ & $p$ \\
\hline 32 & 0 & 0 & 0 & 0 & 0 & 0 & 0 & 0 & 0 & 1 & 64 & 0 & 0 & 0 & 0 & 0 & 0 & 0 & 0 & 0 & 1 \\
\hline 33 & 0 & 0 & 0 & 0 & 0 & 0 & 0 & 0 & 0 & 1 & 65 & 0 & 0 & 0 & 0 & 0 & 0 & 0 & 0 & 0 & 1 \\
\hline 35 & 0 & 0 & 0 & 0 & 0 & 0 & 0 & 0 & 0 & 1 & 67 & 0 & 0 & 0 & 0 & 0 & 0 & 0 & 0 & 0 & 1 \\
\hline 36 & 0 & 0 & 0 & 0 & 0 & 0 & 0 & 0 & 0 & 1 & 68 & 0 & 0 & 0 & 0 & 0 & 0 & 0 & 0 & 0 & 1 \\
\hline 38 & 0 & 0 & 0 & 0 & 0 & 0 & 0 & 0 & 0 & 1 & 70 & 0 & 0 & 0 & 0 & 0 & 0 & 0 & 0 & 0 & 1 \\
\hline 39 & 0 & 0 & 0 & 0 & 0 & 0 & 0 & 0 & 0 & 1 & 71 & 0 & 0 & 0 & 0 & 0 & 0 & 0 & 0 & 0 & 1 \\
\hline 40 & 0 & 0 & 0 & 0 & 0 & 0 & 0 & 0 & 0 & 1 & 72 & 0 & 0 & 0 & 0 & 0 & 0 & 0 & $1<31$ & $\| 1<31$ & 1 \\
\hline 41 & 0 & 0 & 0 & 0 & 0 & 0 & 0 & 0 & 0 & 1 & 73 & 0 & 0 & 0 & 0 & 0 & 0 & 0 & $1<31$ & 0 & $1 / 2$ \\
\hline 45 & 0 & 0 & 0 & 0 & 0 & 0 & 0 & 0 & 0 & 1 & 77 & 0 & 0 & 0 & 0 & 0 & 0 & 0 & $1<31$ & 0 & $1 / 2$ \\
\hline 46 & 0 & 0 & 0 & 0 & 0 & 0 & 0 & 0 & 0 & 1 & 78 & 0 & 0 & 0 & 0 & 0 & 0 & 0 & $1<31$ & 0 & $1 / 2$ \\
\hline 47 & 0 & 0 & 0 & 0 & 0 & 0 & 0 & 0 & 0 & 1 & 79 & 0 & 0 & 0 & 0 & 0 & 0 & 0 & $1<31$ & 0 & $1 / 2$ \\
\hline 48 & 0 & 0 & 0 & 0 & 0 & 0 & 0 & 0 & 0 & 1 & 80 & 0 & 0 & 0 & 0 & 0 & 0 & 0 & 0 & $1<20$ & $1 / 4$ \\
\hline 49 & 0 & 0 & 0 & 0 & 0 & 0 & 0 & 0 & 0 & 1 & 81 & 0 & 0 & 0 & 0 & 0 & 0 & 0 & 0 & 0 & 1 \\
\hline 50 & 0 & 0 & 0 & 0 & 0 & 0 & 0 & 0 & 0 & 1 & 82 & 0 & 0 & 0 & 0 & 0 & 0 & 0 & 0 & 0 & 1 \\
\hline 51 & 0 & 0 & 0 & 0 & 0 & 0 & 0 & 0 & 0 & 1 & 83 & 0 & 0 & 0 & 0 & 0 & 0 & 0 & 0 & 0 & 1 \\
\hline 58 & 0 & $1<31$ & 0 & 0 & 0 & 0 & 0 & 0 & 0 & $1 / 2$ & 90 & 0 & 0 & 0 & 0 & 0 & 0 & 0 & 0 & 0 & 1 \\
\hline 59 & 0 & $1<31$ & 0 & 0 & 0 & 0 & 0 & 0 & 0 & $1 / 2$ & 91 & 0 & 0 & 0 & 0 & 0 & 0 & 0 & 0 & 0 & 1 \\
\hline 60 & 0 & $1<31$ & 0 & 0 & 0 & 0 & 0 & 0 & 0 & $1 / 2$ & 92 & 0 & 0 & 0 & 0 & 0 & 0 & 0 & 0 & 0 & 1 \\
\hline 61 & 0 & $1<31$ & 0 & 0 & 0 & 0 & 0 & 0 & 0 & $1 / 2$ & 93 & 0 & 0 & 0 & 0 & 0 & 0 & 0 & 0 & 0 & 1 \\
\hline 62 & 0 & 0 & 0 & 0 & 0 & 0 & 0 & 0 & $1<20$ & $1 / 4$ & 94 & 0 & 0 & 0 & 0 & 0 & 0 & 0 & 0 & 0 & 1 \\
\hline 63 & 0 & 0 & 0 & 0 & 0 & 0 & 0 & 0 & 0 & 1 & 95 & 0 & 0 & 0 & 0 & 0 & 0 & 0 & 0 & 0 & 1 \\
\hline
\end{tabular}

